\title{
An evaluation method oriented to the comprehensive credibility of simulation data source based on evidence theory
}

\author{
Yanyan Huang*1, Xin $\mathrm{He}^{1}$, Jianyu Wang ${ }^{1}$, Zhen Lei ${ }^{2}$ \\ ${ }^{1}$ School of Automation, Nanjing University of Science and Technology, Nanjing, Jiangsu, China \\ ${ }^{2}$ Department of Scientific Research, Academy of Armored Force Engineering, Beijing, China
}

Received: December 1, 2015

DOI: $10.5430 /$ air.v5n2p32

\author{
Accepted: January 25, 2016 \\ Online Published: February 17, 2016 \\ URL: http://dx.doi.org/10.5430/air.v5n2p32
}

\begin{abstract}
The operational effectiveness of the weapon systems is usually evaluated by the reasonable simulation results. But it is difficult to measure and evaluate the credibility of the simulation data source. An evaluation indices system of the credibility for the simulation data source is built up. A set of quantitative evaluation rules are set up based on the experts' judgments and the fuzzy expression. The credibility is evaluated from the terminal layer indices to the top ones. First, many experts are assigned to evaluate the credibility of the terminal layer indices. Then the credibility of each terminal layer indices of simulation data source is combined and synthesized into the comprehensive credibility of the whole simulation data source. An evaluation method based on the evidence theory is established to integrate above different sub-credibility of simulation data source. All the lower layer indices are required to fuse into the upper ones, and finally get the comprehensive credibility of the simulation data source. For example, the credibility of the simulation data source for the armored equipment systems is evaluated based on above methods. The evaluation results show that the methods based on D-S evidence theory are reasonable and feasible.
\end{abstract}

Key Words: Comprehensive credibility, Simulation data source, Experts' evaluation, Evidence theory

\section{INTRODUCTION}

Operational effectiveness of weapon equipment systems is one of the important indices pursued by both the military and industrial departments. ${ }^{[1-4]}$ The evaluation of the operational effectiveness becomes a key mission for the equipment demonstration and acquisition departments. It is well known that it will cost too much money in doing some tests and experiments for the weapon equipment systems in developing. Hence, the simulation tests are selected to instead of the real tests to support the operational effectiveness evaluation. It is necessary to get more credible and reasonable simulation data to evaluate the weapon system effectiveness. ${ }^{[5-7]}$ It is also required to know and measure the credibility of the simulation data source. As for the credibility evaluation of the simulation data, it is very difficult to get the precise measurements on the credibility of the simulation data source, for the simulation of the combating operation is oriented to the uncertain events in future. The famous work in the simulation field is called VV\&A (validation, verification, accreditation) process, ${ }^{[8-10]}$ which is actually the simulation management process, and it only can ensure the modeling and simulation process in right ways, but it can not measure and evaluate

*Correspondence: Yanyan Huang; Email: huangyy @mail.njust.edu.cn; Address: School of Automation, Nanjing University of Science and Technology, Nanjing 210094, Jiangsu, China. 
the credibility degree of the simulation in quantitative way.

It is necessary to measure and analyze the credibility of the simulation data source so as to support the effectiveness analysis. It is very difficult to measure the credibility of the simulation data source because the credibility is the qualitative index. Usually, the qualitative and quantitative methods are used to solve such kinds of problems. The evaluation method which depends on the experts' experiences is also needed. In fact, if simulation data are accredited by the experienced experts, we can think about the simulation data source having some related credibility. The simulation data collected from simulation test are the terminal layer indices under the evaluation indices systems of the operational effectiveness. Such indices often have lots of information and very clear, so that they are recognized easily by the experts. However, the upper layer indices of the terminal indices are complex and vague, and their credibility is required to accumulate from the credibility of the terminal layer indices. Usually, the credibility of the terminal layer indices can not be sum up directly to the ones of upper layer. How to synthesize and fuse the credibility of lower layer indices into the one of upper layer indices is the main problem to be solved in this paper.

This fusion process of the credibility in lower layer actually is an evaluation process which involved the integration of quantitative and qualitative indices analysis. So far, the experts' evaluation methods and fuzzy logic methods are often used to solve the quantitative and qualitative evaluation question. Although these methods have some subjective moods, they have many well applications. ${ }^{[11,12]}$ About the fusion methods, Dempster-shafer (D-S) theory, ${ }^{[13-18]}$ which can properly describe the degree of belief and extend the subjective probability, has been applied to many fields such as pattern recognition, statistics analysis, and so on. The probability mass functions and Dempster's rules of combination have some references for the work in this paper.

About the organization of this paper, a methodology framework of the credibility evaluation process is put forth; then, under this framework, an evaluation method based on D-S theory and experts' judgments is built to measure and analyze the comprehensive credibility of simulation data source.

\section{A METHOdOLOGY FRAMEWORK OF THE CREDIBILITY EVALUATION PROCESS}

In order to make a clear research roadmap, a methodology framework of the credibility evaluation process is put forth, seeing the Figure 1. In the framework, the part A is about the evaluation of the effectiveness based on the simulation, which mainly depends on the credible simulation results. Part
B is mainly about the credibility evaluation indices systems for the simulation data to be researched. The simulation data are the attributes of the terminal layers indices on the effectiveness evaluation. Hence, we need to build up the comprehensive indices systems for the credibility of simulation data, which are closely related to the evaluation indices systems on effectiveness. Referring to the credibility of the terminal layer indices of the simulation data source, it is necessary to use the experts' experiences and fuzzy logic expressions to quantitatively measure the credibility. What is more, some evaluation rules for the terminal layer indices are also discussed. Part $\mathrm{C}$ mainly includes the methods of measuring for the credibility of the simulation data source. And an evaluation method of comprehensive credibility is built up based on the D-S evidence theory and the quantitative and qualitative analysis by experts.

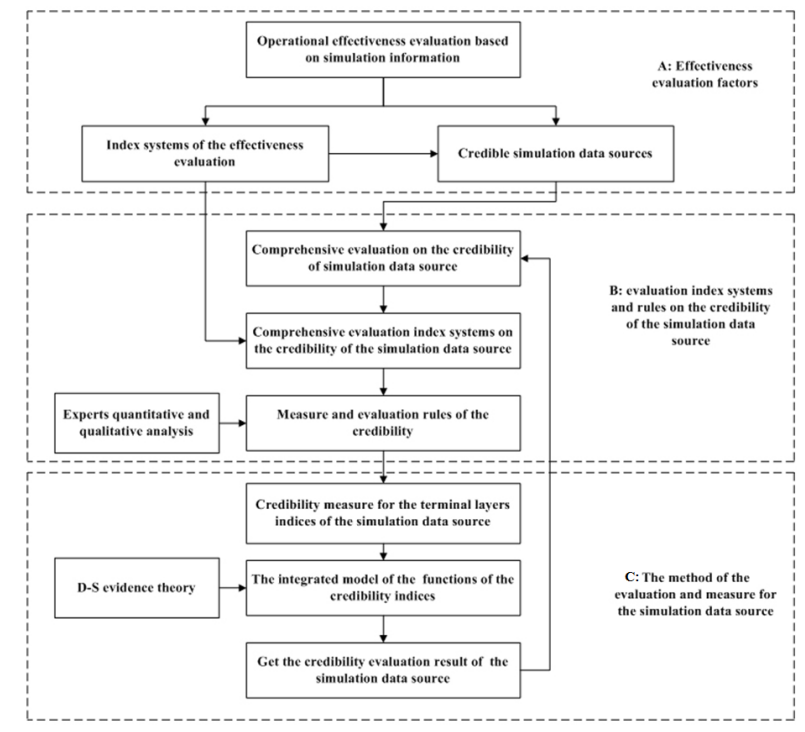

Figure 1. The methodology framework of the credibility evaluation

\section{THE CREDIBILITY EVALUATION PROCESS OF THE SIMULATION DATA SOURCE}

\subsection{The evaluation indices systems on the credibility of simulation data}

Generally, there are a set of evaluation indices systems to evaluate the operational effectiveness based on the simulation. The credibility for the simulation data source is also needed to measure and evaluate. Considering that the simulation data are the terminal indices of the evaluation indices systems of the operational effectiveness, similarly, the evaluation indices systems on the credibility of simulation data are built up, seeing the Figure 2.

Since the terminal layer indices are related to the detailed simulation data, the given experts or evaluation agents can 
judge and analyze them easily. After the credibility of the terminal layer indices is measured, the upper layer indices should be evaluated based on the lower layer indices. The other much upper layer indices can be done in similar way. The top index is the comprehensive credibility of the whole credibility of the simulation data source.

In the Figure 2, the top index, $\mathrm{C}$ is the comprehensive credibility index of the simulation data source; the second layer indices which belong to the top index, such as $S_{1}, S_{r}$, and $S_{m}$, are the sub-indices. In the same way, get the terminal layer indices like $S_{r 1}, S_{r t}$, and $S_{r z}$, which are the detail attributes of the upper indices like Sr. These attributes of terminal indices are closely related to the simulation data. Suppose that there are $n$ evaluation agents (experts), seeing the dished box in the Figure 2, to evaluate the terminal layer indices, and the experts can express their opinions for the terminal layer indices.

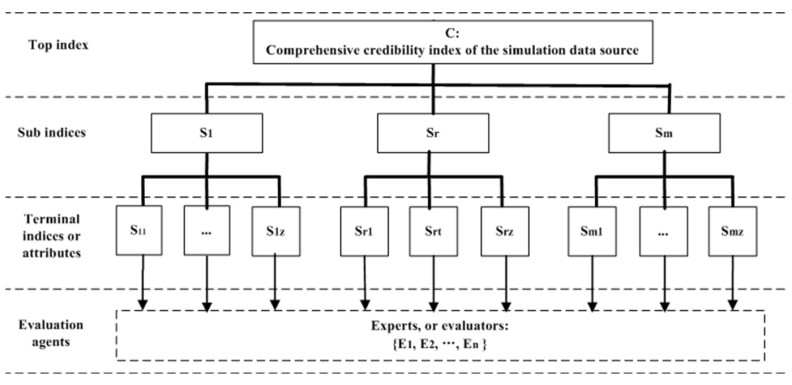

Figure 2. The evaluation indices systems on the credibility of simulation data source

\subsection{Measure and evaluation rules on the credibility ex- pression}

It is necessary to build up a set of rules to measure and evaluate the credibility of the simulation data source. Considering the credibility index is a kind of qualitative index, the experts' experienced information is fit for evaluating the credibility of simulation data. As for the credibility of the upper layer indices of simulation data, it is difficult to measure and evaluate in a precise value. Usually, for such indices, build up the credibility ranking set $\Omega=\left\{A_{1}, A_{2}, A_{3}, A_{4}\right\}$ to describe, and the set $\Omega$ includes four ranking elements: $A_{1}$ (excellent), $A_{2}$ (good), $A_{3}$ (normal), and $A_{4}$ (bad).

Regarding the credibility of the terminal layer indices, it is necessary to use the fuzzy expressions like the "probable/likely" to describe qualitatively the credibility of simulation data. A seven tuples, $U$ is built up to express the credibility degrees from strong to weak, that is, $U=$ certain, likely, very possible, possible, perhaps, somewhat possible, absolute impossible.

In the meantime, provide a seven-parameter vector $\mathrm{V}$ to quantify the elements in $U$ respectively (see Table 1). Such seven-parameter vector $\mathbf{V}$ is described as follows: $\mathbf{V}=$ $\left\{v_{1}, v_{2}, \cdots, v_{7}\right\}$ and $v_{i} \in[0,1]$.

Especially deserving to be mentioned, a formula is put forth to measure the credibility of the simulation data from the simulation alternative $k$, seeing in formula (1).

$$
c_{r t}^{k}=\left\{\begin{array}{c}
v_{1},\left|x_{r t}^{k}-P_{r t}\right| / P_{r t} \in\left[0, \theta_{1}\right], \\
v_{2},\left|x_{r t}^{k}-P_{r t}\right| / P_{r t} \in\left(\theta_{1}, \theta_{2}\right], \\
v_{3},\left|x_{r t}^{k}-P_{r t}\right| / P_{r t} \in\left(\theta_{2}, \theta_{3}\right], \\
v_{4},\left|x_{r t}^{k}-P_{r t}\right| / P_{r t} \in\left(\theta_{3}, \theta_{4}\right], \\
v_{5},\left|x_{r t}^{k}-P_{r t}\right| / P_{r t} \in\left(\theta_{4}, \theta_{5}\right], \\
v_{6},\left|x_{r t}^{k}-P_{r t}\right| / P_{r t} \in\left(\theta_{5}, \theta_{6}\right], \\
v_{7},\left|x_{r t}^{k}-P_{r t}\right| / P_{r t} \in\left[\theta_{6}, \theta_{7}\right] \\
v_{\infty}=0,\left|x_{r t}^{k}-P_{r t}\right| / P_{r t} \in\left(\theta_{7}, \infty\right)
\end{array}\right.
$$

$c_{r t}^{k}$ is the degree value on the th attribute of the $r^{t h}$ index in the simulation alternative $k$, which is one of the elements in the set $V . x_{r t}^{k}$ is the simulation data of the th attribute of the $r^{t h}$ index in the simulation alternative $k ; P_{r t}$ is the given reference value of the $t^{t h}$ attribute of the $r^{t h}$ index; $\theta_{t}$ is the $i^{t h}$ interval value in the seven-tuple $V$. According to above rules, it is very easy to measure and evaluate the indices of the credibility of the simulation data.

Table 1. The quantitative expression of the credibility used by the 7-tuple $V$

\begin{tabular}{llllllll}
\hline $\boldsymbol{\mu}_{\mathbf{j i}}$ & $\mathbf{v}_{\mathbf{1}}$ & $\mathbf{v}_{\mathbf{2}}$ & $\mathbf{v}_{\mathbf{3}}$ & $\mathbf{v}_{\mathbf{4}}$ & $\mathbf{v}_{\mathbf{5}}$ & $\mathbf{v}_{\mathbf{6}}$ & $\mathbf{v}_{\mathbf{7}}$ \\
\hline certain & 1 & 0.75 & 0 & 0 & 0 & 0 & 0 \\
likely & 0.25 & 1 & 0.75 & 0 & 0 & 0 & 0 \\
very possible & 0 & 0.25 & 1 & 0.75 & 0 & 0 & 0 \\
possible & 0 & 0 & 0.5 & 1 & 0.5 & 0 & 0 \\
perhaps & 0 & 0 & 0 & 0.75 & 1 & 0.25 & 0 \\
somewhat possible & 0 & 0 & 0 & 0 & 0.75 & 1 & 0.25 \\
absolute impossible & 0 & 0 & 0 & 0 & 0 & 0.75 & 1 \\
\hline
\end{tabular}

Table 2. The quantitative expression of the credibility ranks based on the seven tuples $V$

\begin{tabular}{llllllll}
\hline $\boldsymbol{\mu}_{\mathbf{j}}\left(\mathbf{A}_{\mathbf{k}}\right)$ & $\mathbf{1}$ & $\mathbf{2}$ & $\mathbf{3}$ & $\mathbf{4}$ & $\mathbf{5}$ & $\mathbf{6}$ & $\mathbf{7}$ \\
\hline excellent $\left(\mathrm{A}_{1}\right)$ & 1 & 0.75 & 0 & 0 & 0 & 0 & 0 \\
$\operatorname{good}\left(\mathrm{A}_{2}\right)$ & 0 & 0.25 & 0.75 & 0.5 & 0 & 0 & 0 \\
$\operatorname{normal}\left(\mathrm{A}_{3}\right)$ & 0 & 0 & 0 & 0.75 & 1 & 0.25 & 0 \\
$\operatorname{bad}\left(\mathrm{A}_{4}\right)$ & 0 & 0 & 0 & 0 & 0 & 0.75 & 1 \\
\hline
\end{tabular}

On the basis of the expression of the credibility ranks, the fuzzy membership function (like subjective probability) of the index $S_{r t}$ evaluated by the expert $j$ is defined in (2): 


$$
\mu_{j}^{\left(s_{r t}\right)^{*}}\left(A_{k}\right)=\frac{\sum_{i=1}^{7}\left(\mu_{j i}^{\left(s_{r t}\right)} \wedge \mu_{i}\left(A_{k}\right)\right)}{\sum_{i=1}^{7}\left(\mu_{j i}^{\left(s_{r t}\right)} \vee \mu_{i}\left(A_{k}\right)\right)}
$$

Where, $k=1,2,3,4, j=1,2, \cdots, n$. $\wedge$ is minimizing operator; $\vee$ is maximizing operator. The value of the (2) is the matching degree of the $S_{r t}$ comparing with all the credibility ranks. Having, $0 \leq \mu_{j}^{\left(S_{r t}\right)^{*}} \leq 1$. Because the experts' expression is no the exclusiveness, the matching degree value is not always equal to 1 . That is to say, $\sum_{k=1}^{4} \mu_{j}^{\left(S_{r t}\right)^{*}}\left(A_{k}\right) \neq 1$, so it is required to make the following normalization.

$$
\mu_{j}^{\left(s_{r t}\right)}\left(A_{k}\right)=\frac{\mu_{j}^{\left(s_{r t}\right)^{*}}\left(A_{k}\right)}{\sum_{k=1}^{4} \mu_{j}^{\left(s_{r t}\right)^{*}}\left(A_{k}\right)}
$$

Where, $k=1,2,3,4, j=1,2, \cdots, n, \mu_{j}^{S_{r t}}\left(A_{k}\right)$ is described as the evaluation value of the expert $j$ belonging to all elements of the $\Omega$. It can be expressed in the (4).

$$
Q_{j}^{\left(s_{r t}\right)}=\left\{\mu_{j}^{\left(s_{r t}\right)}\left(A_{1}\right), \mu_{j}^{\left(s_{r t}\right)}\left(A_{2}\right), \mu_{j}^{\left(s_{r t}\right)}\left(A_{3}\right), \mu_{j}^{\left(s_{r t}\right)}\left(A_{4}\right)\right\}
$$

\subsection{The experts' opinions expression and the mass func- tions construction}

For the difference in the knowledge, understanding and the preference, the reliability of opinion from each expert may be different. For the index $S_{r t}$, it is necessary to give the weights of the experts as follows.

$$
\left(\varpi_{1}^{\left(s_{r n}\right)}, \varpi_{2}^{\left(s_{r t}\right)}, \ldots, \varpi_{n}^{\left(s_{n}\right)}\right), \text { and } \sum_{k=1}^{n} \varpi_{j}^{\left(s_{r t}\right)}=1
$$

Given a group of experts, it is supposed that the higher the weight of the expert, the more reliable his opinion is. The reliability of the expert $j$ giving to the index $S_{r t}$ is described as below:

$$
\alpha_{j}^{\left(s_{r t}\right)}=\gamma_{j}^{\left(s_{r t}\right)} \cdot \frac{\varpi_{j}^{\left(s_{r t}\right)}}{\max _{1 \leq i \leq n} \varpi_{j}^{\left(s_{r t}\right)}}
$$

Where, $j=1,2, \cdots, n ; \gamma_{j}^{\left(S_{r t}\right)}$ is a coefficient about the preference of the experts $j$, let $0.9 \leq \gamma_{j}^{\left(S_{r t}\right)} \leq 1$. And thus, from (6), gets the result: $0 \leq \alpha_{j}^{\left(S_{r t}\right)} \leq 1$. As for the index $S_{r t}$, the revised evaluation values of the expert $j$ belonging to all elements of the $\Omega$ is described in (7).

$$
Q_{j}^{\left(s_{r f}\right)^{*}}=\alpha_{j}^{\left(s_{r t}\right)} \cdot Q_{j}^{\left(s_{r f}\right)}
$$

Obviously, $\sum_{k=1}^{4} \alpha_{j}^{\left(S_{r t}\right)} \mu_{j}^{\left(S_{r t}\right)}\left(A_{k}\right) \leq 1$, and the uncertain part of the evaluation value about the expert $j$ belonging to all elements of the $\Omega$ is $1-\sum_{k=1}^{4} \alpha_{j}^{S_{r t}} \mu_{j}^{S_{r t}}\left(A_{k}\right)$.

Based on the D-S evidence theory, the expression in (7) has met the conditions of the mass function. And therefore, the new mass function about the evaluation value to the $S_{r t}$ from the expert $j$ is defined as follows:

$$
m_{j}^{\left(s_{r t}\right)}(A)= \begin{cases}\alpha_{j}^{\left(s_{r t}\right)} \cdot \mu_{j}^{\left(s_{r t}\right)}\left(A_{1}\right) & A=\left\{A_{1}\right\} \\ \alpha_{j}^{\left(s_{r t}\right)} \cdot \mu_{j}^{\left(s_{r t}\right)}\left(A_{2}\right) & A=\left\{A_{2}\right\} \\ \alpha_{j}^{\left(s_{r t}\right)} \cdot \mu_{j}^{\left(s_{r t}\right)}\left(A_{3}\right) & A=\left\{A_{3}\right\} \\ \alpha_{j}^{\left(s_{r t}\right)} \cdot \mu_{j}^{\left(s_{r t}\right)}\left(A_{4}\right) & A=\left\{A_{4}\right\} \\ 1-\sum_{k=1}^{4} \alpha_{j}^{\left(s_{r t}\right)} \cdot \mu_{j}^{\left(s_{r t}\right)}\left(A_{1}\right) & A=\Omega\end{cases}
$$

It also can be written for short in (9).

$$
m_{j}^{\left(s_{t f}\right)}=\left(m_{j}^{\left(s_{f t}\right)}\left(A_{1}\right), m_{j}^{\left(s_{s_{t}}\right)}\left(A_{2}\right), m_{j}^{\left(s_{s_{t}}\right)}\left(A_{3}\right), m_{j}^{\left(s_{r_{f}}\right)}\left(A_{4}\right), m_{j}^{\left(s_{s_{f}}\right)}(\Omega)\right)
$$

Where, $j=1,2, \cdots, n ; r=1,2, \cdots, m ; t=1,2, \cdots, z$. By means of the mass function, the credibility degree distribution of the index $S_{r t}$ by the expert $j$ can be reflected properly.

\subsection{Synthesis method of the credibility indices}

Considering the uncertain part of the credibility indices, it is necessary to use a proper method to integrate the subcredibility of the terminal layer indices into the ones of the upper layer indices. Because the mass functions for the credibility of the terminal indices are constructed in above charters, it is convenient to use the Dempster's rule of combination, seeing in formula (10), to make a comprehensive measurement and integration evaluation.

Dempster's rule of combination(or evidential reasoning): if the $m_{1}, m_{2}, \cdots, m_{n}$ are mass functions, then the synthesis function $m(\cdot)$ is also a mass function.

$$
\begin{aligned}
& m(\varphi)=0, \\
& \mathrm{~m}(\mathrm{~A})=\mathrm{m}_{1}(\mathrm{~A}) \oplus \mathrm{m}_{2}(\mathrm{~A}) \oplus \ldots \oplus \mathrm{m}_{\mathrm{n}}(\mathrm{A}) \\
& =k \sum_{n A_{j}=\phi 1 \leq i \leq n} m_{i}\left(A_{j}\right), \quad A \neq \varnothing \\
& k^{-1}=1-\sum_{\cap A_{j}=\varnothing 1 \leq i \leq n} \prod_{i} m_{i}\left(A_{j}\right)=\sum_{n A_{j} \neq \varnothing \varnothing 1 \leq i \leq n} m_{i}\left(A_{j}\right)
\end{aligned}
$$

Where, if $k^{-1}=0$, it shows that there are conflicts between the $m_{i}$. The parameter $k$ describes the conflict degree of the mass functions; the evidential reasoning formula can not work under the $n$ complete conflict evidences. The synthesis results of evidential reasoning are to reduce the uncertainty 
information of the credibility; in the meanwhile, the new mass function will be produced to synthesize the evidences of the upper layer index.

There are $n$ experts in the evaluation, so that there are $n$ pieces of opinions in form of the mass function to the evaluation index. The synthesis result of these mass functions is following:

$$
m^{\left(s_{t}\right)}=\left(m^{\left(s_{t}\right)}\left(A_{1}\right), m^{\left(s_{t}\right)}\left(A_{2}\right), m^{\left(s_{t}\right)}\left(A_{3}\right), m^{\left(s_{t}\right)}\left(A_{4}\right), m^{\left(s_{t}\right)}(\Omega)\right)
$$

Where $m^{\left(S_{r t}\right)}\left(A_{i}\right)$ is the credibility degree of $s_{r t}$ comparing with rank $A_{i}$ based on the synthesis opinions of all the experts.

After finish the synthesis of the each of the indices in the terminal layer, $z$ mass functions are gotten respectively, they are $m^{\left(s_{r 1}\right)}, m^{\left(s_{r 2}\right)}, \cdots, m^{\left(s_{r z}\right)}$. The weights of the $\mathrm{z}$ indices, such as $w^{\left(s_{r 1}\right)}, w^{\left(s_{r 2}\right)}, \cdots, w^{\left(s_{r z}\right)}$, are used to describe the importance directly comparing to the upper index. But these weights are not wholly represented for the importance comparing to the top index. Hence, the comparative importance degree is defined as follows:

$$
\beta_{s_{r t}}=\zeta \cdot \frac{\omega^{\left(s_{r t}\right)}}{\max _{1 \leq t \leq z} \omega^{\left(s_{r t}\right)}}
$$

Where, $\zeta$ is a correction coefficient, and it is related to the experts' experiences, Usually, $0.9 \leq \zeta \leq 1$. So there is the following result: $0.9 \leq \beta_{S_{r t}} \leq 1,(t=1,2, \cdots, z)$. For the uncertainty of the weights of indices, the correction mass function for the index $s_{r t}$ can be revised as following description (12).

$$
m^{\left(s_{r t}\right) *}=\beta_{s_{r t}} \cdot m^{\left(s_{r t}\right)}
$$

Deduced from formula $12, \sum_{k=1}^{4} \beta_{S_{r t}} \cdot m^{\left(S_{r t}\right)}\left(A_{k}\right) \leq 1$, while the uncertain part for the srt by the expert $j$ is $1-\sum_{k=1}^{4} \beta_{S_{r t}} \cdot m^{\left(S_{r t}\right)}\left(A_{k}\right)$. By means of the comparative importance degree, all the correction mass functions of $z$ indices are gotten as follows: $m^{\left(S_{r 1}\right)^{*}}, m^{\left(S_{r 2}\right)^{*}}, m^{\left(S_{r z}\right)^{*}}$.

Using the D-S formula again, the mass function of the upper layer index $s_{r}$ is obtained by synthesizing the $z$ mass functions of the terminal layer indices.

$$
m^{\left(s_{r}\right)}=\left(m^{\left(s_{r}\right)}\left(A_{1}\right), m^{\left(s_{r}\right)}\left(A_{2}\right), m^{\left(s_{r}\right)}\left(A_{3}\right), m^{\left(s_{r}\right)}\left(A_{4}\right), m^{\left(s_{r}\right)}(\Omega)\right)
$$

The synthesis mass functions of other upper indices may be deduced by similar way to the top index. As a result, for the simulation alternative $\mathrm{F}$, the synthesis mass function which integrates the credibility indices from bottom to top in order is gotten in the end as follows:

$$
m^{(F)}=\left(m^{(F)}\left(A_{1}\right), m^{(F)}\left(A_{2}\right), m^{(F)}\left(A_{3}\right), m^{(F)}\left(A_{4}\right)\right)
$$

The top index mass function $m^{(F)}$ is the comprehensive credibility expression of all the indices systems for the simulation alternative $\mathrm{F}$, and it is the very measurement and evaluation that the comprehensive credibility of all the simulation data in alternative $\mathrm{F}$, which compares with the given credibility ranks set $\Omega$. Considering the elements of the credibility ranks $\Omega$, there is a coefficient $d_{i}$ for each rank $A_{i}, i=1,2, \cdots, 5$.

Finally, the comprehensive credibility of the simulation data in alternative $\mathrm{F}$ can be measured in (15).

$c^{(F)}(A)=d_{1} * m^{(F)}\left(A_{1}\right)+d_{2} * m^{(F)}\left(A_{2}\right)+d_{3} * m^{(F)}\left(A_{3}\right)+d_{4} * m^{(F)}\left(A_{4}\right)+d_{5} * m^{(F)}(\Omega)$

\section{AN APPLication EXAMPLE}

In this paper, take the operational effectiveness evaluation based on the simulation of the armored equipment systems for example. By means of the operational simulation, a batch of simulation data is generated to support the evaluation of the operational effectiveness of the armored equipment systems. It is necessary to measure the credibility of such a batch of simulation data. Noticing in this paper, we are not going to talk about the detailed system of modeling and simulation, but our main object is to research the method how to measure and evaluate such simulation data sources.

With the above mentioned method, the application example is carried out as follows. Firstly, build up the evaluation indices systems on the credibility of simulation data, as shown in Figure 3. The index systems are based on the operational effectiveness evaluation indices systems. The terminal layer indices are about the original data from simulation application. The credibility evaluation will start from the bottom to top.

Here 5 experts are selected as the evaluation group, and they give the credibility opinions for the terminal layer indices, and synthesize these credibility degrees based on the evidential reasoning.

Take example for the indices like $S_{21}, S_{22}$, and $S_{23}$, which are under the index $S_{2}$, their terminal indices values are the mean values from three simulation tests results, seeing the Table 3. For these terminal indices, usually, we can give pre-judgment values as the references in Table 4.

The experts can give the credibility preferences for the indices based on the simulation data and the references values, 
the evaluation moods expression like the "probable/likely" are used to express the qualitative credibility of simulation data source. The seven tuples $\mathrm{U}$ is used to describe the degrees of credibility of the index $S_{2 i}$, as shown in Table 5 .

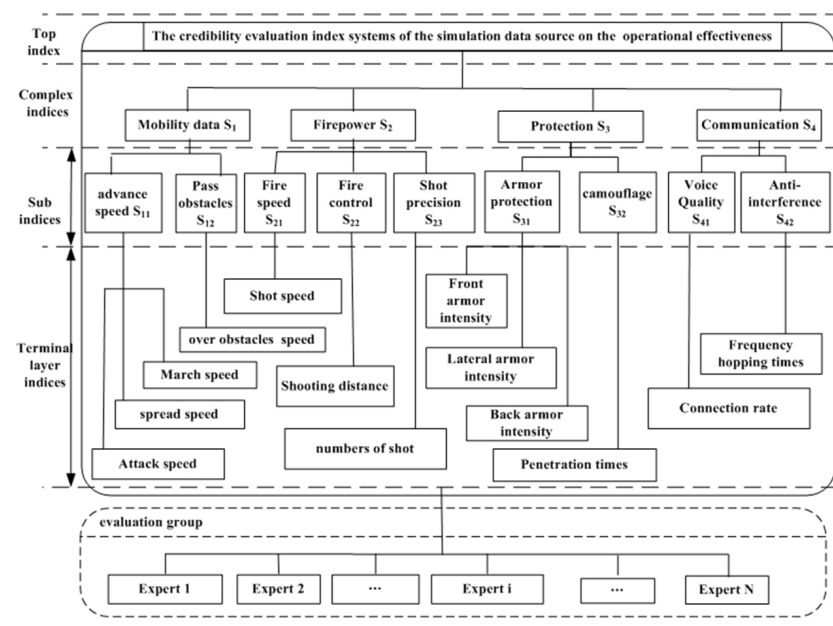

Figure 3. The credibility evaluation indices systems of the simulation data on the operational effectiveness

Table 3. Simulation data of the indices of the upper index $S_{2}$

\begin{tabular}{llll}
\hline $\begin{array}{l}\text { Simulation test } \\
\text { order } \mathbf{i}\end{array}$ & $\mathbf{S}_{\mathbf{2 1}}$ (time) & $\mathbf{S}_{\mathbf{2 2}}(\mathbf{k m})$ & $\mathbf{S}_{\mathbf{2 3}}$ (time) \\
\hline 1 & 2 & 1,800 & 1 \\
2 & 5 & 1,700 & 3 \\
3 & 2 & 1,900 & 2 \\
Mean value & 3 & 1,800 & 2 \\
\hline
\end{tabular}

Table 4. Pre-judgment values of the indices

\begin{tabular}{llll}
\hline $\begin{array}{l}\text { Pre-judgment } \\
\text { index }\end{array}$ & $\mathbf{S}_{\mathbf{2 1}}$ (time) & $\mathbf{S}_{\mathbf{2 2}}(\mathbf{k m})$ & $\mathbf{S}_{\mathbf{2 3}}$ (time) \\
\hline Value & 5 & 1,850 & 3 \\
\hline
\end{tabular}

Table 5. The experts' evaluation opinions for the indices $s_{2}$

\begin{tabular}{llll}
\hline expert $\mathbf{i}$ & $\mathbf{S}_{21}$ & $\mathbf{S}_{22}$ & $\mathbf{S}_{23}$ \\
\hline expert 1 & likely & very possible & likely \\
expert 2 & somewhat possible & perhaps & very possible \\
expert 3 & perhaps & very possible & likely \\
expert 4 & somewhat possible & somewhat possible & perhaps \\
expert 5 & perhaps & perhaps & somewhat \\
& & possible \\
\hline
\end{tabular}

For instance, about the index $S_{22}$, every expert will give an evaluation expression for it. The expert 1 give the fuzzy credibility for the $S_{22}$ comparing with all the elements in Published by Sciedu Press the credibility ranks $\Omega$, and the membership degrees can be listed in the Table 6 .

Table 6. The fuzzy credibility for index $s_{22}$ by expert 1 and the related operators

\begin{tabular}{llllllll}
\hline $\begin{array}{l}\text { v3="very } \\
\text { possible" }\end{array}$ & $\mathbf{0}$ & $\mathbf{0 . 2 5}$ & $\mathbf{1}$ & $\mathbf{0 . 7 5}$ & $\mathbf{0}$ & $\mathbf{0}$ & $\mathbf{0}$ \\
\hline excellent $\left(\mathrm{A}_{1}\right)$ & 1 & 0.75 & 0 & 0 & 0 & 0 & 0 \\
$\operatorname{good}\left(\mathrm{A}_{2}\right)$ & 0 & 0.25 & 0.75 & 0.5 & 0 & 0 & 0 \\
$\operatorname{normal}\left(\mathrm{A}_{3}\right)$ & 0 & 0 & 0 & 0.75 & 1 & 0.25 & 0 \\
$\operatorname{bad}\left(\mathrm{A}_{4}\right)$ & 0 & 0 & 0 & 0 & 0 & 0.75 & 1 \\
$\mathrm{~V}_{3} \wedge \mathrm{A}_{1}$ & 0 & 0.25 & 0 & 0 & 0 & 0 & 0 \\
$\mathrm{~V}_{3} \vee \mathrm{A}_{1}$ & 1 & 0.75 & 1 & 0.75 & 0 & 0 & 0 \\
$\mathrm{~V}_{3} \wedge \mathrm{A}_{2}$ & 0 & 0.25 & 0.75 & 0.5 & 0 & 0 & 0 \\
$\mathrm{~V}_{3} \vee \mathrm{A}_{2}$ & 0 & 0.75 & 1 & 0.75 & 0 & 0 & 0 \\
$\mathrm{~V}_{3} \wedge \mathrm{A}_{3}$ & 0 & 0 & 0 & 0.75 & 0 & 0 & 0 \\
$\mathrm{~V}_{3} \vee \mathrm{A}_{3}$ & 0 & 0.25 & 1 & 0.75 & 1 & 0.25 & 0 \\
$\mathrm{~V}_{3} \wedge \mathrm{A}_{4}$ & 0 & 0 & 0 & 0 & 0 & 0 & 0 \\
$\mathrm{~V}_{3} \vee A_{4}$ & 0 & 0.25 & 1 & 0.75 & 0 & 0.75 & 1 \\
\hline & & & & & & & \\
& & & & &
\end{tabular}

In the Table $6, \wedge$ is minimizing operator; $\vee$ is maximizing operator. Calculate the membership degrees of the index $S_{22}$ based on the (2).

$$
\begin{gathered}
\mu_{1}^{\left(s_{22}\right) *}\left(A_{1}\right)=\frac{\sum_{i=1}^{7}\left(\mu_{1 i}^{\left(s_{22}\right)} \wedge \mu_{i}\left(A_{1}\right)\right)}{\sum_{i=1}^{7}\left(\mu_{1 i}^{\left(s_{22}\right)} \vee \mu_{i}\left(A_{1}\right)\right)}=0.5 / 3.5=0.0714 ; \\
\mu_{1}^{\left(s_{22}\right) *}\left(A_{2}\right)=1.5 / 2.5=0.6 ; \mu_{1}^{\left(s_{22}\right) *}\left(A_{3}\right)=0.75 / 3.25=0.2308 ; \mu_{1}^{\left(s_{22}\right) *}\left(A_{3}\right)=0 .
\end{gathered}
$$

Normalize the membership degrees, and get the expert 1 opinion belonging to each of the credibility ranks $\Omega$.

$\mu_{1}^{\left(s_{22}\right)}\left(A_{1}\right)=0.0792 ; \mu_{1}^{\left(s_{22}\right)}\left(A_{2}\right)=0.665 ; \mu_{1}^{\left(s_{22}\right)}\left(A_{3}\right)=0.2558 ; \mu_{1}^{\left(s_{22}\right)}\left(A_{4}\right)=0$

Simplify the expression of the above membership degrees in formula (4).

$Q_{1}^{\left(s_{22}\right)}=\{0.0792,0.665,0.2558,0\}$

In the same way, the other four experts' opinions belonging to the credibility ranks $\Omega$ are gotten as follows.

$$
\begin{aligned}
& Q_{2}^{\left(s_{22}\right)}=\{0,0.4681,0.5319,0\} \\
& Q_{3}^{\left(s_{22}\right)}=\{0.0792,0.665,0.2558,0\} \\
& Q_{4}^{\left(s_{22}\right)}=\{0,0.1346,0.8077,0.0577\} \\
& Q_{5}^{\left(s_{22}\right)}=\{0,0.4681,0.5319,0\}
\end{aligned}
$$

The weights of the 5 experts can be calculated by the AHP (analytic hierarchy process), and here it does not need to repeat in detail.

$\left(\varpi_{1}^{\left(s_{r t}\right)}, \varpi_{2}^{\left(s_{r t}\right)}, \ldots, \varpi_{5}^{\left(s_{r t}\right)}\right)=(0.21,0.2,0.18,0.19,0.22)$ 
Set $\gamma_{j}^{\left(s_{22}\right)}=0.92$, and get the comparative reliability of each expert as follows.

$\left\{\alpha_{1}^{\left(s_{22}\right)}, \alpha_{2}^{\left(s_{22}\right)}, \ldots, \alpha_{5}^{\left(s_{22}\right)}\right\}=(0.879,0.836,0.753,0.795,0.92)$

In light of the (7), build up the mass functions of the credibility index by means of the five experts. For example, the $S_{22}$ is measured by the expert 1 , and get the following mass function.

$$
\begin{aligned}
m_{1}^{\left(s_{22}\right)} & =\left(m_{1}^{\left(s_{22}\right)}\left(A_{1}\right), m_{1}^{\left(s_{22}\right)}\left(A_{2}\right), m_{1}^{\left(s_{22}\right)}\left(A_{3}\right), m_{1}^{\left(s_{22}\right)}\left(A_{4}\right), m_{1}^{\left(s_{22}\right)}(\Omega)\right) \\
& =(0.0696,0.585,0.2248,0,0.1206)
\end{aligned}
$$

The other 4 mass functions are measured by the other four experts respectively.

$$
\begin{aligned}
& m_{2}^{\left(s_{22}\right)}=(0.0662,0.556,0.188,0,0.190) \\
& m_{3}^{\left(s_{22}\right)}=(0.0596,0.501,0.1926,0,0.2468) \\
& m_{4}^{\left(s_{22}\right)}=(0.0630,0.529,0.2034,0,0.2046) \\
& m_{5}^{\left(s_{22}\right)}=(0.0729,0.6118,0.2353,0,0.08)
\end{aligned}
$$

Synthesize the above 5 mass functions in the (9) based on evidential reasoning, and get the comprehensive credibility

\begin{tabular}{|c|c|c|c|c|c|}
\hline$T_{i j}$ & $\begin{array}{l}m_{2}^{\left(S_{22}\right)}\left(A_{1}\right) \\
=0.0662\end{array}$ & $\begin{array}{l}m_{2}^{\left(S_{22}\right)}\left(A_{2}\right) \\
=0.556\end{array}$ & $\begin{array}{l}m_{2}^{\left(S_{22}\right)}\left(A_{3}\right) \\
=0.188\end{array}$ & $\begin{array}{l}m_{2}^{\left(S_{22}\right)}\left(A_{4}\right) \\
=0\end{array}$ & $\begin{array}{l}m_{2}^{\left(S_{22}\right)}(\Omega) \\
=0.190\end{array}$ \\
\hline $\begin{array}{l}m_{1}^{\left(s_{22}\right)}\left(A_{1}\right) \\
=0.0696\end{array}$ & 0.0046 & 0 & 0 & 0 & 0.0138 \\
\hline $\begin{array}{l}m_{1}^{\left(s_{22}\right)}\left(A_{2}\right) \\
=0.585\end{array}$ & 0 & 0.3253 & 0 & 0 & 0.1112 \\
\hline $\begin{array}{l}m_{1}^{\left(s_{22}\right)}\left(A_{3}\right) \\
=0.2248\end{array}$ & 0 & 0 & 0.0423 & 0 & 0.0427 \\
\hline $\begin{array}{l}m_{1}^{\left(s_{22}\right)}\left(A_{4}\right) \\
=0\end{array}$ & 0 & 0 & 0 & 0 & 0 \\
\hline $\begin{array}{l}m_{1}^{\left(s_{22}\right)}(\Omega) \\
=0.1206\end{array}$ & 0.008 & 0.0671 & 0.0227 & 0 & 0.0229 \\
\hline
\end{tabular}
mass function of the $s_{22}$. It is necessary to make the direct sum between the mass functions, like $m_{1}^{\left(s_{22}\right)} \bigoplus m_{2}^{\left(s_{22}\right)}$. The results of the direct sum are shown in Table 7.

Table 7. The direct sum of two mass functions by the expert 1 and expert 2

Where

$$
\begin{aligned}
& \mathrm{T}_{11}\left(\mathrm{~A}_{1}\right)=m_{1}^{\left(s_{22}\right)}\left(A_{1}\right) m_{2}^{\left(s_{22}\right)}\left(A_{1}\right)=0.0046, \mathrm{~T}_{15}\left(\mathrm{~A}_{1}\right)=m_{1}^{\left(s_{22}\right)}\left(A_{1}\right) m_{2}^{\left(s_{22}\right)}(\Omega)=0.0138, \\
& M_{1}^{\left(s_{22}\right)}\left(A_{1}\right)=m_{1} \oplus m_{2}\left(A_{1}\right)=0.0046+0.0138+0.008=0.0264 \\
& M_{1}^{\left(s_{22}\right)}\left(A_{2}\right)=m_{1} \oplus m_{2}\left(A_{2}\right)=0.3253+0.1112+0.0671=0.5036 \\
& M_{1}^{\left(s_{22}\right)}\left(A_{3}\right)=m_{1} \oplus m_{2}\left(A_{3}\right)=0.0423+0.0427+0.0227=0.1077 \\
& M_{1}^{\left(s_{22}\right)}\left(A_{4}\right)=m_{1} \oplus m_{2}\left(A_{4}\right)=0 \\
& M_{1}^{\left(s_{22}\right)}(\Omega)=m_{1} \oplus m_{2}(\Omega)=0.0229
\end{aligned}
$$

Due to, let $X=\left\{A_{i}\right\}, Y=\left\{A_{i}\right\}, i=1,2,3,4$.

$$
\mathrm{k}^{-1}=1-\sum_{X \cap Y=\phi} m_{1}^{\left(S_{22}\right)}(X) m_{2}^{\left(S_{22}\right)}(Y)=1-0.3394=0.6606
$$

Normalize the $M_{1}^{\left(s_{22}\right)}\left(A_{i}\right)$, and get:

$$
\begin{aligned}
& \left(M_{1}^{\left(s_{22}\right)}\left(A_{1}\right), M_{1}^{\left(s_{22}\right)}\left(A_{2}\right), M_{1}^{\left(s_{22}\right)}\left(A_{3}\right), M_{1}^{\left(s_{22}\right)}\left(A_{4}\right), M_{1}^{\left(s_{22}\right)}(\Omega)\right) \\
& =(0.04,0.762,0.163,0,0.0351)
\end{aligned}
$$

Then, $M_{1}^{\left(s_{22}\right)}\left(A_{i}\right) \bigoplus m_{3}^{\left(s_{22}\right)}\left(A_{i}\right)$, and get the $M_{2}^{\left(s_{22}\right)}\left(A_{i}\right)$, till gets: $M_{4}^{\left(s_{22}\right)}\left(A_{i}\right) \bigoplus m_{5}^{\left(s_{22}\right)}\left(A_{i}\right)$.

As a result, $m^{\left(s_{22}\right)}=(0.0059,0.8642,0.1290,0.0,0.0009)$.

In the same way of the index $S_{22}$, the deduced results of the $S_{21}$, and $S_{23}$ are gotten as follows.

$$
\begin{aligned}
& m^{\left(s_{21}\right)}=(0.0067,0.7340,0.2093,0.0045,0.0455) \\
& m^{\left(s_{23}\right)}=(0.0033,0.8905,0.0914,0,0.0148)
\end{aligned}
$$

Given the relative weights of the $S_{21}, S_{22}$ and $S_{23}$ are listed successively like $0.32,0.38,0.30$. Set $\zeta_{s_{2 i}}(i=1,2,3)$ is 0.93 ,then use (11), get $\beta_{s_{2 i}}=(0.783,0.93,0.734)$.

Further, combine with the relative weights to revise the three indices, and get the revised mass functions:

$m^{\left(s_{22}\right)}=(0.0046,0.6767,0.101,0.0,0.2177)$

$m^{\left(s_{21}\right)}=(0.0062,0.683,0.195,0.0042,0.1123)$

$m^{\left(s_{23}\right)}=(0.0024,0.6536,0.0671,0,0.2769)$

Synthesize the mass functions of $S_{21}, S_{22}$ and $S_{23}$, and get the mass function of the supper layer index $S_{2}$.

$m^{\left(S_{2}\right)}=m^{\left(S_{21}\right)} \oplus m^{\left(S_{22}\right)} \oplus m^{\left(S_{23}\right)}=(0.0013,0.7932,0.1887,0,0.0168)$

Similar to above steps, the mass functions like $m^{\left(S_{1}\right)}, m^{\left(S_{3}\right)}$ and $m^{\left(S_{4}\right)}$ are calculated. Given the comparative importance degrees of the $S_{1}, S_{2}$ and $S_{4}$ and the coefficients $\beta_{s_{i}}(i=1,2,3,4)$, it is easy to synthesize the 4 mass functions into the mass function of the top index, which stands for the credibility of the whole simulation data source. $m^{(S)}=(0.0008($ excellent $), 0.701($ good $), 0.2021($ normal $)$, 0.0352(bad), 0.05(unknown)).

Obviously, the credibility of the whole simulation data source in alternative $S$ is mainly described in the "good" rank with 0.701 reliability degree.

Finally, combining with the coefficients $D=\left\{d_{i}\right\}=$ $(1,0.8,0.6,0.4,0.0)$ and the formula (15), the comprehensive credibility of the simulation data in alternative $\mathrm{F}$ ( $\mathrm{F}$ is a simulation alternative) is measured as follows. 
$C^{(F)}(A)=0.0008 * 1+0.701 * 0.8+0.2021 * 0.6+0.0352 *$

$0.4+0.05 * 0.0=0.6969$

This means the comprehensive credibility of the simulation data source from the simulation alternative $\mathrm{F}$ is up to 0.6969 ; such credibility is in line with the actual actions of the simulation systems in the operational effectiveness evaluation.

\section{Conclusion AND FUTURE WORK}

Aiming at the credibility measure and evaluation of the simulation data source, by means of the expert expression and fuzzy language, and a set of evaluation method based on the D-S evidence theory is established to research. According to such methods, take the operational effectiveness simulation of the armored equipment systems as example, combining with experts' expression and the evaluation rules, the credibility of the terminal layer indices of the simulation data sources are measured; after the credibility mass functions on the terminal layer indices are constructed, then synthesize such mass functions in indices order from lower to the top based on evidential reasoning theory. Finally, the comprehensive credibility is gotten, and the evaluation results show that the methods in this paper are applicable and feasible. About the future work, we need to make further research on the evaluation method on the credibility of the simulation data source, and the there are several advice:

(1) Continue to complete the evaluation indices systems of the credibility of the simulation data source. It is helpful to use OODA(observe, orient,decide,act) loop to build the evaluation indices systems for the weapon operation effectiveness. ${ }^{[19]}$

(2) Continue to complete the evaluation method based on the D-S evidence theory, and especially, make a short, concise, and easy-using evaluation method for the public user.

(3) Build up an evaluation system in software based on the evaluation method discussing in this paper so as to make easily to test the simulation data source.

\section{ACKNOWLEDgements}

This work has been supported by Natural Science Foundation of China (61374186, 60804035).

\section{REFERENCES}

[1] Yáñez J, Ortuño T, Vitoriano B. A simulation approach to reliability analysis of weapon systems. European Journal of Operational Research. 1997 July; 100(1): 216-224. http://dx.doi.org/10.10 16/S0377-2217 (97) 00274-9

[2] Monnin M, Iung B, Sénéchal O. Dynamic behavioural model for assessing impact of regeneration actions on system availability: Application to weapon systems. Reliability Engineering \& System Safety. 2011 March; 96(3): 410-24.

[3] Jiang J, Li X, Zhou ZJ, et al. Weapon System Capability Assessment under uncertainty based on the evidential reasoning approach. Expert Systems with Applications. 2011 October; 38(11): 13773-84.

[4] Zhao QS, Chang LL, Zeng P, et al. Problem Frame Analysis of Weapon System of Systems Requirement. Procedia Engineering. 2011; 15: 1466-70. http://dx.doi.org/10.1016/j.proeng. 2011.08 .272

[5] Zhou SY, Wu WH, Zhou SM, et al. A New Situation Assessment Model for Modern Within-Visual-Range Air Combat. Procedia Engineering. 2012; 29: 339-43.

[6] Walker JD. Weapon system effectiveness. Aerospace America. 2001 December; 39(12): 93-98.

[7] Fortanbary MW, Mansager BK, Newberry CF. Supporting acquisition decisions through effective experimental design. Mathematical and Computer Modelling. 1996 January; 23(1-2): 81-90. http: //dx.doi.org/10.1016/0895-7177 (95) 00219-7

[8] BALCI O. Verification,Validation, and Certification of Modeling and Simulation Applications. In: Proceedings of the 2003 winter simulation Conference, Chick S, Sanchez PJ, eds., New Orleans, LA; 2003 Dec. 7-10. p. 150-8.

[9] CARSON JS. Model verification and Validation. In: Proceeding of the 34th winter simulation conference, Yucesan E, Chen $\mathrm{CH}$, Snowdon JL, eds., San Diego; 2002 Dec. 8-11. p.52-8.
[10] SARGENT SR. Verification and Validation of Simulation Models. In: Proceedings of the 2007 Winter Simulation Conference, eds. Henderson SG, Biller B, etc. Washington, DC; 2007 Dec. 9-12. p.124-37.

[11] Zadeh LA. A theory of approximate reasoning. Machine Intelligence, Ellis Horwood, Chichester, U.K.; 1979. p. 149-94.

[12] Kandel A. Fuzzy expert systems, CRC Press; 1991. p. 1-336.

[13] Dempster A. Upper and lower probabilities induced by a multivalued mapping. Annals of Mathematical Statistics. 1967; 38: 325-39. http://dx.doi.org/10.1214/aoms/1177698950

[14] Dempster A, Kong A. Uncertain evidence and artificial analysis Journal of Statistical Planning and Inference. 1988; 20: 355-68. http://dx.doi.org/10.1016/0378-3758(88)90097-3

[15] Shafer G. A Mathematical Theory of Evidence. Princeton University Press. Princeton. NJ; 1976.

[16] Shafer G, Shenoy PP, Mellouli K. Propgation belief functions in qualitative Markov trees. International Journal of Approximate Reasoning. 1987; 1(4): 349-400. http://dx.doi .org/10.1016/088 8-613X (87) 90024-7

[17] Jones RW, Lowe A, Harrison MJ. A framework for intelligent medical diagnosis using the theory of evidence. Knowledge-Based Systems. 2002; 15(1): 77-84. http://dx.doi.org/10.1016/S0950-705 1 (01) 00123-X

[18] Chen SW, Liu J, Wang H, et al. A group decision making model for partially ordered preference under uncertainty. Information Fusion. 2015; 25: 32-41. http://dx.doi.org/10.1016/j.inffus.20 14.11 .001

[19] Huang YY. Modeling and simulation method of the emergency response systems based on OODA. Journal of Knowledge-Based Systems. 2015; 89: 527-40. http://dx.doi.org/10.1016/j.knosy s. 2015.08 .020 